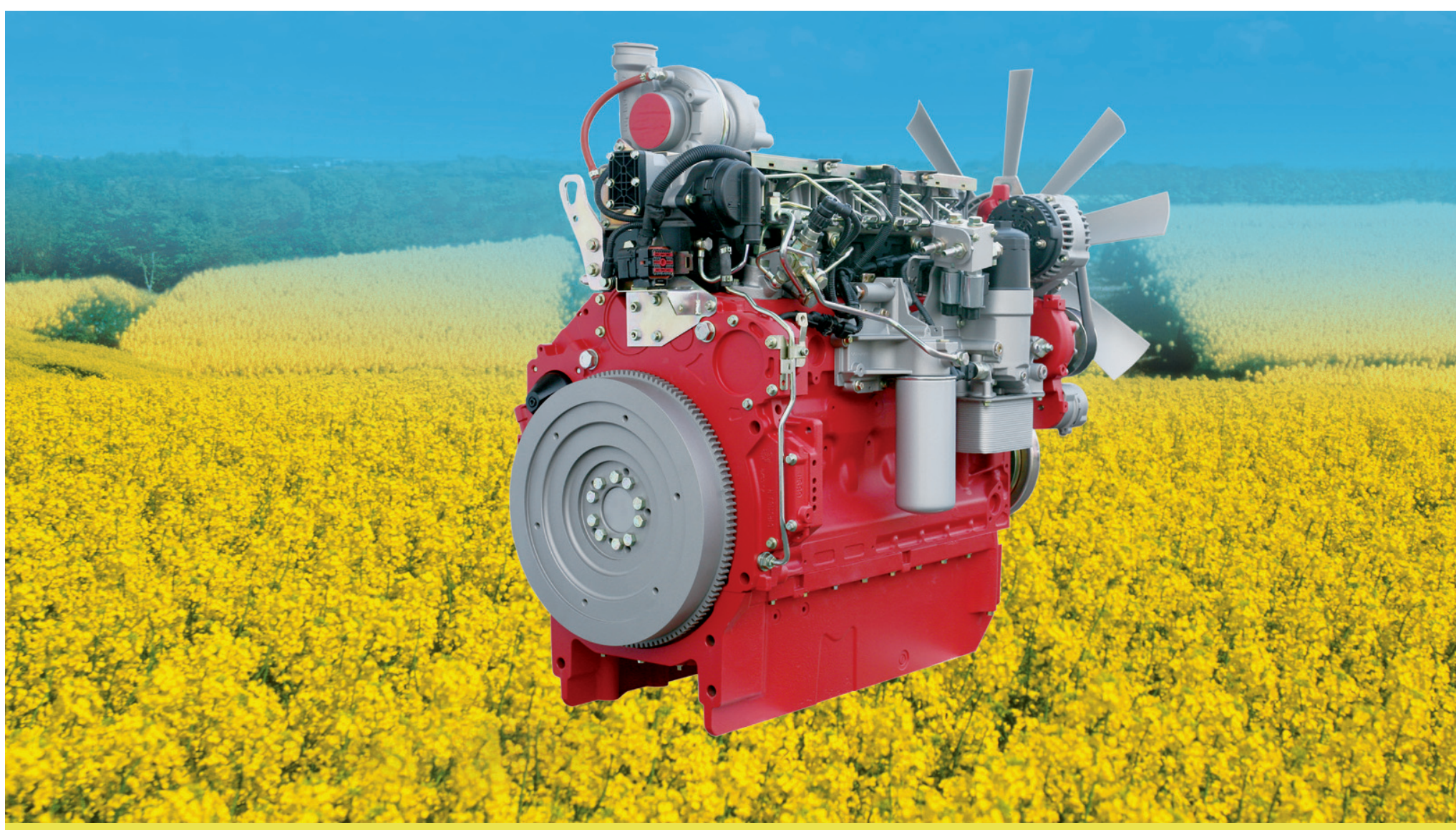

\title{
ELEMENTBELASTUNGEN VON ABGASNACHBEHANDLUNGSSYSTEMEN DURCH BIODIESEL
}

Gerade für den Landtechnikbereich ist Biodiesel als Kraftstoff attraktiv. Mit der Einführung neuer Emissionsstufen ist der Einsatz von Abgasnachbehandlungssystemen auch bei diesen Anwendungen unumgänglich. Der Betrieb mit Biodiesel ist dann allerdings problematisch, weil Biodiesel Spuren anorganischer Elemente enthält. Sie bilden bei der Verbrennung im Motor Rückstände, welche die Komponenten für die Abgasnachbehandlung dauerhaft schädigen können. Deutz und ASG haben die Auswirkungen aktueller Biodieselqualitäten auf die Systeme im realen Betrieb untersucht. 


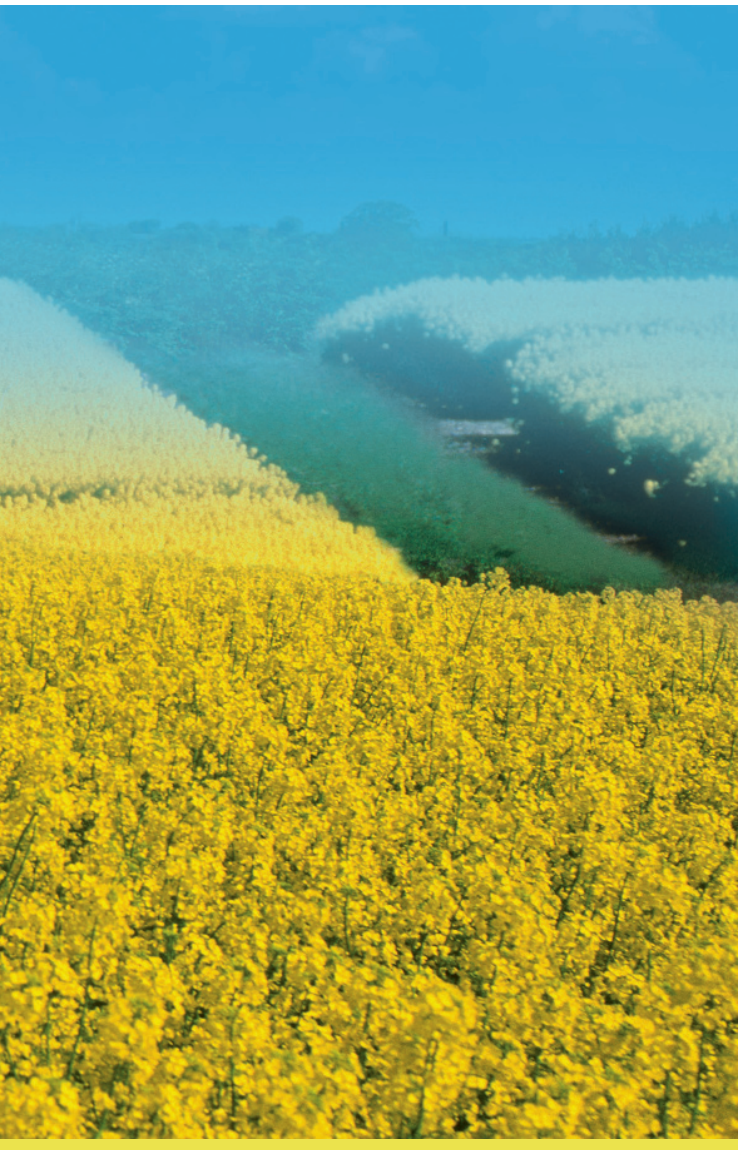

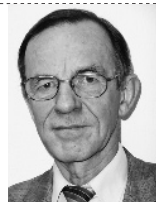

DR.-ING. HANS-WALTER KNUTH

ist ehemaliger Teamleiter Abgas und Betriebsstoffe bei der Deutz AG in Köln.

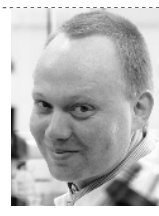

DR. RER. NAT. HENDRIK STEIN

ist Leiter der Prozessentwicklung und stellvertretender Laborleiter bei der ASG Analytik-Service Gesellschaft mbH in Neusäß.

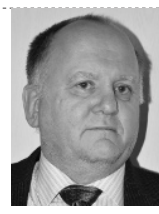

DR, RER, NAT. THOMAS WILHARM ist Geschäftsführer der ASG AnalytikService Gesellschaft mbH in Neusäß.

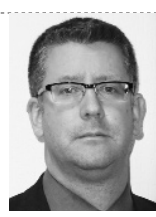

DIPL.-ING. MARKUS WINKLER

ist verantwortlich für die Betriebsstoffanalytik und Betriebsstofffreigaben bei der Deutz AG in Köln.

\section{MOTIVATION}

Die Substitution mineralölbasierter Kraftstoffe durch Biokraftstoffe ist eine Maßnahme zur Realisierung der politischen Ziele, die $\mathrm{CO}_{2}$-Emissionen deutlich zu reduzieren und fossile Ressourcen zu schonen. Gestützt durch das Europäische Gemeinschaftsrecht der Kraftstoffqualitätsrichtlinie (2003/30/EG) und der Erneuerbare-Energien-Richtlinie (2009/28/EG) verfolgt die EU-Kommission mit ihrem Aktionsplan zur Förderung der Verwendung von erneuerbaren Energien im Verkehrssektor das spezifische Ziel, bis zum Jahr 2020 mindestens 10 \% der europaweit eingesetzten konventionellen Kraftstoffe durch Kraftstoffe aus regenerativen Quellen zu ersetzen. Diese Richtlinien sind Teil des europäischen Klima- und Energiepakets, das der Europäische Rat im Dezember 2008 beschlossen hatte. Die EU-Mitgliedsstaaten haben anerkannt, dass dieses Ziel vorrangig nur mit Biokraftstoffen erreicht werden kann, indem sie ihre nationalen Aktionspläne der Kommission übermittelt haben [1].

Aus Sicht des Motorherstellers Deutz besitzt Biodiesel einige Kraftstoffeigenschaften, welche mit potenziell negativen Auswirkungen auf das Einspritzund das Schmierölsystem des Motors verbunden sind. Bei Beachtung von Vorsichtsmaßnahmen können Motoren aber trotzdem zuverlässig mit reinem Biodiesel betrieben werden. So hat Deutz eine Vielzahl von Motorbaureihen für den Biodieseleinsatz freigegeben [2]. Gerade für den Landtechnikbereich können Biodieselfreigaben aufgrund steuerlicher Vorteile attraktiv sein.

Mit sich verschärfenden Abgasgrenzwerten für Heavy-Duty-Dieselmotoren können diese Anforderungen nur noch mit dem Einsatz von Abgasnachbehandlungsmaßnahmen (beispielsweise Dieselpartikelfilter und/oder selektive katalytische Reduktion (SCR)) erfüllt werden. Der Einsatz von Biodiesel in Kombination mit diesen Nachbehandlungstechniken ist insofern mit zusätzlichen Problemen behaftet, als Biodiesel in Spuren anorganische Elemente wie Natrium, Kalium, Calcium und Magnesium enthält. Anorganische Elemente bilden bei der Verbrennung im Motor Aschen (zum Beispiel Oxide), welche zum Teil in die Komponenten der Nachbehandlung eingetragen werden und ihre Wirksamkeit dauerhaft schädigen können. Die in der europäischen Norm 
Jahresmittelwerte und präzisierte Jahresmittelwerte für die Summengehalte an Alkalimetallen in Biodiesel

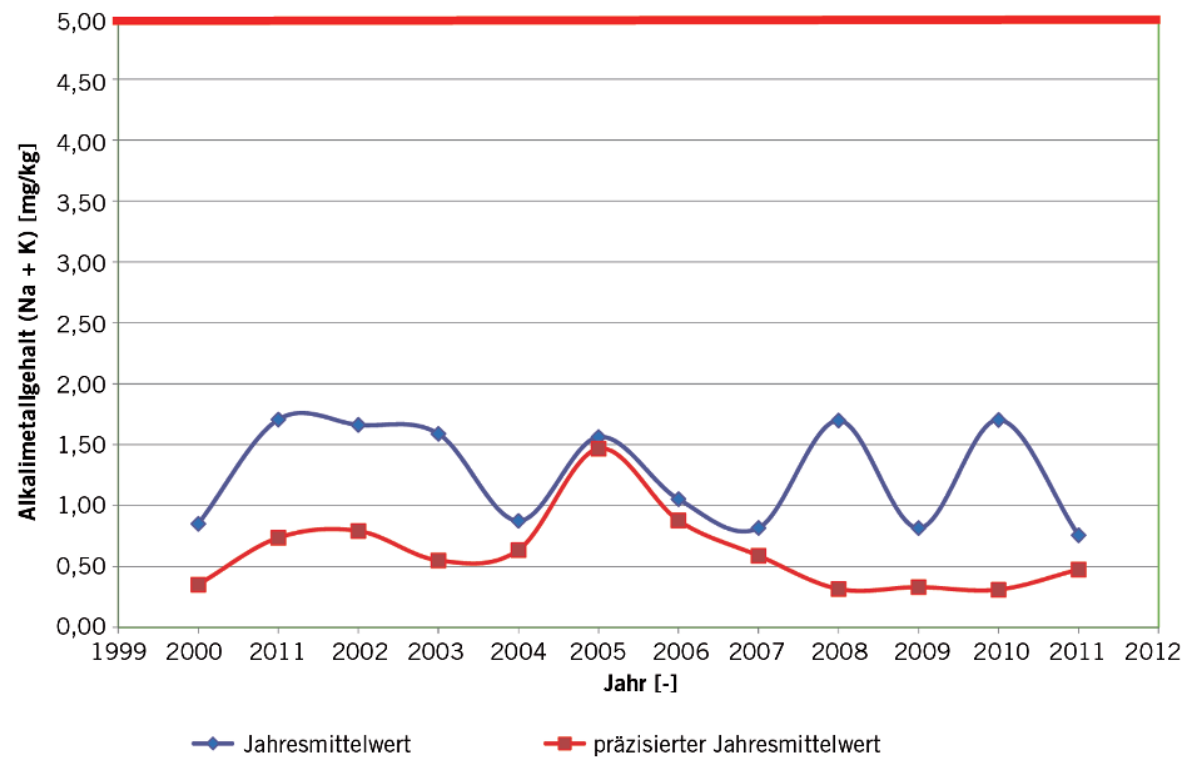

(2) Jahresmittelwerte und präzisierte Jahresmittelwerte für die Summengehalte an Erdalkalimetallen in Biodiesel

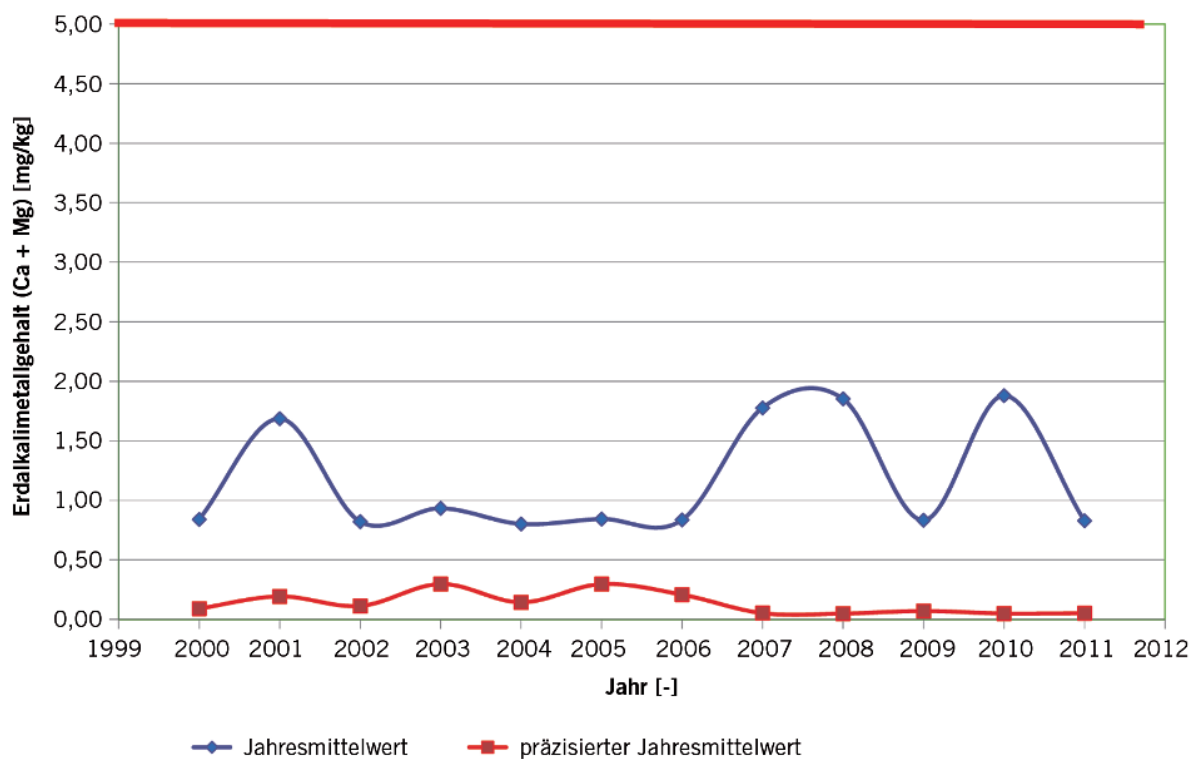

$\leadsto$ Jahresmittelwert $\quad \rightarrow$ präzisierter Jahresmittelwert

für Biodiesel DIN EN 14214 [3] vorgegebenen Grenzwerte für anorganische Elemente (in Summe maximal $5 \mathrm{mg} / \mathrm{kg}$ für $\mathrm{Na}+\mathrm{K}$, maximal $5 \mathrm{mg} / \mathrm{kg}$ für $\mathrm{Ca}+\mathrm{Mg}$ ) liegen dabei in einem Bereich, der nach dem bisherigen Stand der Technik zu einer irreversiblen Schädigung der Nachbehandlungskomponenten führen würde, sollten diese Grenzwerte tatsächlich die heute im Feld zu beobachtende Biodieselqualität widerspiegeln.

Um Entwicklern von Motoren und Abgasnachbehandlungssystemen reali- tätsnahe Daten zur Abschätzung der potenziellen Frachten von Aschebildnern und Katalysatorgiften bereitstellen zu können, wurden im Rahmen einer von der Union zur Förderung der Oel- und Proteinpflanzen e.V. (UFOP) geförderten Studie [4] Analyseergebnisse marktrelevanter Biodieselmuster aus den Jahren 2000 bis 2011 ausgewertet. Als Datenbasis dienten die Datenbanken der Arbeitsgemeinschaft Qualitätsmanagement Biodiesel e.V. (AGQM) und der Analytik-Service $\mathrm{GmbH}$ (ASG).

\section{STAND DER AKTUELLEN MOTOR- UND ABGAS - NACHBEHANDLUNGSTECHNIK}

Durch die drastischen Verschärfungen der Abgasgesetzgebung in Europa wurden in den letzten 20 Jahren die Grenzwerte für Stickoxide $\left(\mathrm{NO}_{\mathrm{x}}\right)$ um circa $97 \%$ und für die Partikelmasse um circa 98,5 \% reduziert. Bei schweren Nutzfahrzeugen hat sich schon mit Einführung der Abgasstufe Euro IV SCR als die führende Abgasnachbehandlungstechnik durchgesetzt. Die Definition eines Grenzwerts für die Partikelanzahl ab Euro VI wird eine Kombination aus SCR-System und Partikelfilter erforderlich machen.

Während bei mobilen Arbeitsmaschinen die seit Januar 2006 geltende Emissionsstufe IIIA mit rein innermotorischen Maßnahmen erfüllt werden konnte, zum Beispiel durch Ladeluftkühlung, Abgasrückführung und Steigerung des Einspritzdrucks, ist mit der Abgasstufe IIIB seit 2011 zusätzlich der Einsatz von Abgasnachbehandlungssystemen unumgänglich. Deutz hat sich hierbei für die folgenden Technikpfade entschieden:

: Partikelfilter in Kombination mit Abgasrückführung für Baumaschinen und andere Industrieanwendungen : SCR (Vanadium-Wolfram-Titandioxid, VWT) ohne Abgasrückführung für Landtechnikanwendungen.

Für Landtechnikanwendungen wurde die SCR-Technik ausgewählt, weil in diesem Markt Anforderungen hinsichtlich eines besonders niedrigen Kraftstoffverbrauchs bestehen. Da die Stickoxide mit der SCR-Technik effektiv abgesenkt werden, kann der Motor durch die Applikation eines früheren Einspritzzeitpunkts verbrauchsoptimal eingestellt werden.

\section{BIODIESELUNTERSUCHUNG}

In den vergangenen elf Jahren wurden von der Arbeitsgemeinschaft Qualitätsmanagement Biodiesel (AGQM) durch unangekündigte Kontrollen bei Biodieselherstellern mehr als 8000 Analysedaten von Phosphor-, und Metallgehalten in Biodiesel gesammelt. Die Elementgehalte werden dabei mit dem Verfahren der ICP OES (optische Spektralanalyse mit induktiv gekoppeltem Plasma) bestimmt. Alkali- und Erdalkalimetalle werden mit der Prüfmethode DIN EN 14538 [5] gemessen, der Phosphorgehalt mit dem Verfahren DIN EN 14107 [6]. 
(3) Motoren, Abgasnachbehandlungssysteme und Schmieröle aus den beiden Biodieselfeldtests als Basis für die Berechnungen

\begin{tabular}{|c|c|c|c|c|}
\hline \multicolumn{5}{|c|}{ BIODIESEL-FELDTEST 2008/2009 (UFOP-PROJEKT 540/80) } \\
\hline MOTOR & LEISTUNG / DREHMOMENT & HUBRAUM & ABGASSTUFE & ÖLQUALITÄT \\
\hline $\begin{array}{l}\text { TDC2013 } \\
\text { L04 4V }\end{array}$ & $\begin{array}{l}158 \mathrm{~kW} \text { bei } 2300 / \min / \\
\text { MDmax: } 800 \mathrm{Nm}\end{array}$ & 4,81 & \multirow{2}{*}{$\begin{array}{l}\text { Euro IV/V } \\
\text { mit VWT-SCR }\end{array}$} & \multirow{7}{*}{$\begin{array}{l}\text { ACEA E7 } \\
\text { DQC III-10 } \\
\text { Elementgehalte } \\
\text { für Vergiftungs- } \\
\text { berechnung: } \\
\text { Ca: } 3350 \mathrm{mg} / \mathrm{kg} \\
\text { Mg: } 10 \mathrm{mg} / \mathrm{kg} \\
\text { P: } 1300 \mathrm{mg} / \mathrm{kg} \\
\text { Zn: } 1400 \mathrm{mg} / \mathrm{kg}\end{array}$} \\
\hline $\begin{array}{l}\text { TDC2013 } \\
\text { L06 4V }\end{array}$ & $\begin{array}{l}235 \mathrm{~kW} \text { bei } 2300 / \mathrm{min} / \\
\text { MDmax: } 1200 \mathrm{Nm}\end{array}$ & 7,21 & & \\
\hline \multicolumn{4}{|c|}{ BIODIESEL-FELDTEST 2010/2011 (UFOP-PROJEKT 540/103) } & \\
\hline MOTOR & LEISTUNG / DREHMOMENT & HUBRAUM & ABGASSTUFE & \\
\hline TDC 7.8 L6 & 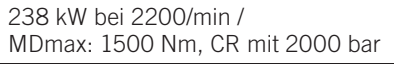 & 7,81 & \multirow{3}{*}{$\begin{array}{l}\text { Stufe } \\
\text { COM IIIB mit } \\
\text { VWT-SCR }\end{array}$} & \\
\hline TDC 6.1 L6 & 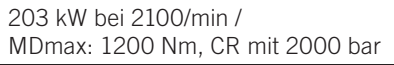 & 6,11 & & \\
\hline TDC 6.1 L6 & 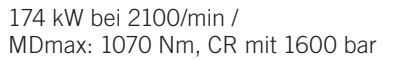 & 6,11 & & \\
\hline
\end{tabular}

In einem ersten Schritt wurden Jahresmittelwerte berechnet, die alle Prüfergebnisse unterhalb der Bestimmungsgrenzen (zum Beispiel <0,5 mg/kg) unberücksichtigt ließen. In einem zweiten Schritt wurden auch diese Daten integriert. Dies erfolgte auf der Basis von Nachauswertungen der Rohdaten und sorgte somit für eine Präzisierung der Jahresmittelwerte. $(1)$ und 2 zeigen die errechneten Ergebnisse für die Summengehalte an Alkaliund Erdalkalielementen für die Jahre 2000 bis 2011. Am oberen Ende der Skala ist der gemäß DIN EN 14214 zulässige Höchstwert von $5 \mathrm{mg} / \mathrm{kg}$ für die Summengehalte an Alkali- beziehungsweise Erdalkalimetallgehalten abgebildet.

Alkalimetalle wie Natrium und Kalium werden durch die Umesterungskatalysatoren während des Biodieselherstellungsprozesses in den Kraftstoff eingetragen. Demgegenüber stammen die Erdalkalimetalle Calcium und Magnesium im Wesentlichen aus dem zur Herstellung verwendeten Pflanzenöl, beziehungsweise können bei der Biodieselwäsche durch das Waschwasser eingetragen werden. Grundsätzlich bewegen sich die errechneten Jahresmittelwerte deutlich unterhalb der jeweiligen Normgrenzwerte. Tendenziell finden sich mehr Alkali- als Erdalkalimetalle im Biodiesel. Die präzisierten Mittelwerte unterschreiten immer die zugehörigen Jahresmittelwerte.

\section{AUSWIRKUNG VON ASCHEAKKU- MULATION AUF DIE ABGAS- NACHBEHANDLUNGSSYSTEME}

In der Literatur wird die Vergiftung von SCR-Katalysatoren durch Metalle umfangreich beschrieben, und gerade basische Elemente wie Kalium und Natrium zeigen einen besonders hohen Vergiftungseffekt [7-10]. Die Vergiftung beruht darauf, dass die sauren Reaktionszentren des SCR-Katalysators neutralisiert werden und somit für die Aufnahme des ebenfalls basischen Ammoniaks nicht mehr zur Verfügung stehen. Die Folge ist eine Wirkungsgradverschlechterung bis hin zu einer völligen Deaktivierung. Bereits 0,1 Massen-\% Kalium im Washcoat eines VanadiumSCR-Katalysators führen entsprechend den Untersuchungsergebnissen eines FVV-Projekts [7] zu einer Wirkungsgradverschlechterung von circa $20 \%$. Natrium ist ein ähnlich starkes Gift, die Erdalkalimetalle Calcium und Magnesium haben meist eine geringere, aber immer noch erhebliche Vergiftungswirkung.

Auf Basis der in der Literatur verfügbaren Vergiftungsdaten wurde eine rechnerische Methode zur Abschätzung der Vergiftung eines VWT-SCR-Katalysators, wie sie bei Verbrennung von Biodiesel mit definierten Elementgehalten zu erwarten ist, entwickelt. Zusätzlich wurde auch die Vergiftung durch Elemente betrachtet, die aus dem Schmieröl aufgrund des Ölverbrauchs resultieren. Der Ölverbrauch beträgt zwar nur circa 0,05 \% des Kraftstoffverbrauchs, da aber im Schmieröl wesentlich höhere Elementkonzentrationen vorliegen, ist auch die Vergiftungsgefahr durch Schmierölbestandteile nicht zu vernachlässigen. Hier eine kurze Beschreibung der Berechnungsmethode für die SCR-Vergiftung:

: Berechnung der Elementemission aus Kraftstoffverbrauch und Ölverbrauch sowie den Elementgehalten in Biodiesel und Schmieröl (Daten aus $\mathbf{3}$ bei

\section{WTZ Roßlau \\ Wissenschaftlich-Technisches Zentrum für Motoren- und Maschinenforschung Roßlau gGmbH}

Ankündigung und Aufruf zur Vortragseinreichung

\section{Dessauer Gasmotoren- Konferenz}

Dessau-Roßlau, 21. und 22. März 2013

Wir möchten Ihnen die 8. DessauerGasmotoren-Konferenz mit dem Aufruf zur Einreichung von Vortragsthemen ankündigen.

\section{Themenschwerpunkte}

- zukünftige Gasmotorenkonzepte

- alle Arten von Gasmotoren für den Transport- und Industriesektor

- Aspekte der Konstruktion und Entwicklung von Gasmotoren und Gasmotorenzubehör

- Zündsysteme, zentrale und zylinderselektive Gemischbildung, Motorauslegung, -steuerung, -überwachung, -simulation und -diagnose

- alternative gasförmige Kraftstoffe z. B Wasserstoff, DME LPG

- Zuverlässigkeit, Betreibererfahrungen.

In einer konferenzbegleitenden Ausstellung haben Sie die Möglichkeit, Ihr Unternehmen sowie Produkte und Leistungen vorzustellen.

\section{Termine}

15. August 2012

Termin für die Einreichung der Kurzfassung

\section{September 2012}

Bestätigung der Vortragsannahme

\section{Dezember 2012}

Abgabetermin der druckfähigen

Vortragsfassung

21. - 22. März 2013

Konferenz

Weitere Informationen über die Kontaktadresse

Mühlenreihe 2a

D-06862 Dessau-Roßlau

Frau Constanze Jackowski

Tel.: $\quad+4934901883-124$

Fax: $\quad+4934901883-120$

E-Mail: Jackowski@wtz.de

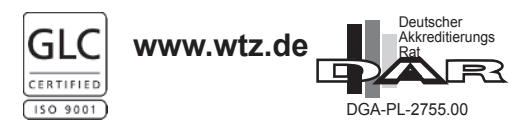




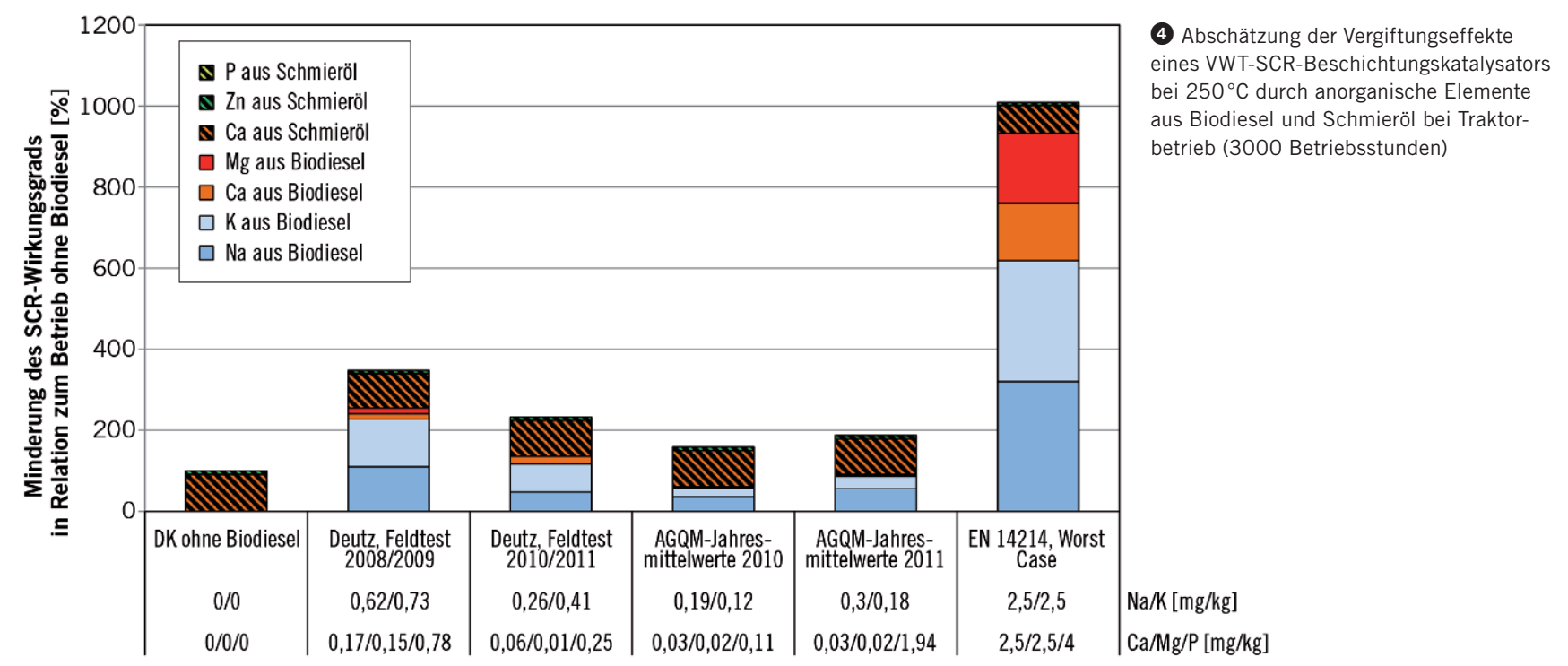

einer Annahme von $30 \%$ des Ölverbrauchs durch Verdampfung, das heißt ohne Elementemission)

: Berechnung der Elementkonzentrationen im Washcoat als Funktion der Laufzeit bei Annahme einer definierten Depositionsrate $(2,5 \%$, ermittelt auf Basis von Washcoat-Analysen aus dem Feldtestprojekt)

: Berechnung der Vergiftung durch Verwendung von elementspezifischen Vergiftungsraten aus dem FVV-Projekt [7] und Summation über alle Elemente.

4 zeigt die berechneten VWT-SCR-Wirkungsgradminderungen für verschiedene Biodieselqualitäten hinsichtlich Elementverschmutzungen (100\% entsprechen Dieselkraftstoff ohne Biodie- sel). Würden die Biodieselproduzenten tatsächlich grenzwertigen Biodiesel nach DIN EN 14214 herstellen, würde ein neunfach erhöhter und somit wesentlich zu hoher Vergiftungseffekt eintreten, der einen Einsatz von Biodiesel in Motoren mit SCR-Abgasnachbehandlung unmöglich machen würde. Auf Basis der AGQMDaten aus dem Jahr 2010 sowie der DeutzFeldtestdaten im Rahmen des UFOP-Projekts 540/103 von 2010 bis 2011 hingegen ergibt sich eine Vergiftung durch Biodiesel, die nur leicht über der Vergiftung durch Schmieröl liegt. Bei der Nachvermessung des Wirkungsgrads der SCRKatalysatoren aus den beiden Feldtests wurde eine im Vergleich zu den Rechnungen geringere Wirkungsgradminde- rung ermittelt $[11,12]$. Aufgrund der vorliegenden Ergebnisse hat sich Deutz entschlossen, Motoren der Stufe IIIB mit SCR-Abgasnachbehandlungssystem für den Betrieb mit B100 freizugeben. Dies erfolgt allerdings unter der Voraussetzung, dass der SCR-Katalysator bei Biodieselbetrieb alle $3000 \mathrm{~h}$ getauscht wird.

Ein ähnliches Berechnungsverfahren wurde für die Akkumulation von Aschen in Partikelfiltern angewendet. Hierbei wurde von einer Retentionsrate von $100 \%$ ausgegangen. Die Ergebnisse dieser Berechnung für den gleichen Einsatzfall wie oben zeigt $\mathbf{5}$. Auch hier entsprechen $100 \%$ einem Dieselkraftstoff ohne Biodiesel. Die Relation der schädigenden Effekte der verschiedenen Kraftstoffqua-

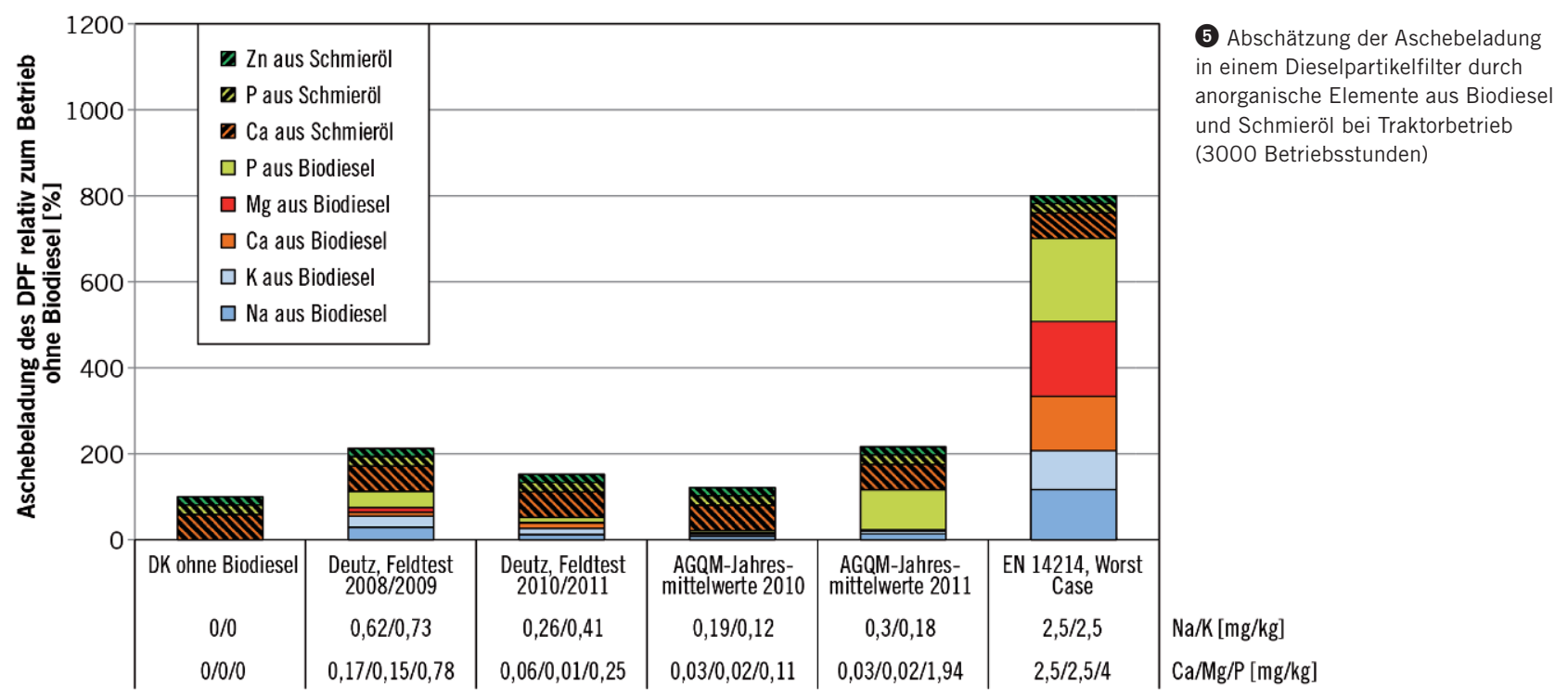


litäten ist bei einer Aschebeladung sehr ähnlich wie bei einer SCR-Vergiftung. Ein (hypothetischer) grenzwertiger Biodiesel nach DIN EN 14214 hätte eine elffach höhere Ascheakkumulation wie ein biodieselfreier Dieselkraftstoff und würde den Filter in weniger als 500 n verstopfen. Dies wird mit der Einführung von Euro VI ab 2014 relevant sein, weil dann Partikelfilter und SCR-Katalysatoren eingesetzt werden - auch im Landtechnikbereich. Auch bezüglich der Ascheakkumulation ist zu konstatieren, dass die realen Biodieselqualitäten ein wesentlich besseres Bild abgeben als eine Worst-Case-Qualität, welche die Spezifikationsgrenzen ausschöpft.

\section{ZUSAMMENFASSUNG UND AUSBLICK}

Die Auswertung der AQGM- und Feldtestdaten zeigt, dass die Biodieselqualität im Feld deutlich besser ist als es die Normgrenzwerte zulassen. Ähnliche Ergebnisse liefern zwei Studien aus den USA [13, 14]. In diesem Zusammenhang ist es sehr zu begrüßen, dass die AGQM die Ergebnisse ihrer unangemeldeten Qualitätsüberwachung nunmehr jährlich publiziert. Die niedrigen Elementkonzentrationen sind ein wichtiger Baustein für die Freigabe der Deutz-Landtechnikmotoren der EU-Stufe IIIB.

Bei zukünftigen Euro-IV-Motorkonzepten und der damit verbundenen Anwendung anderer Abgasnachbehandlungstechniken (Fe-Zeolith-SCR-Substrate, DOC) ist eine grundlegende Neubewertung der Biodieselfreigaben erforderlich. Wegen der Vergiftungsproblematik besteht aus Sicht der Motorhersteller dringender Handlungsbedarf, die Grenzwerte der EN 14214 erheblich abzusenken und entsprechende Analysenmethoden zu entwickeln. Bereits umgesetzt wurden Maßnahmen zur Absenkung der Elementgehalte im Schmieröl. Auch die Maßnahmen der Motorhersteller zur generellen Absenkung des Schmierölverbrauchs sind weitestgehend ausgereizt.

\section{LITERATURHINWEISE}

[1] http://ec.europa.eu/energy/renewables/transparency_platform/doc/national_renewable_energy_ action_plan_germany_de.pdf

[2] Technisches Rundschreiben Kraftstoffe. TR 0199-99-1218, Deutz AG, Köln

[3] DIN EN 14214: Kraftstoffe für Kraftfahrzeuge Fettsäure-Methylester (FAME) für Dieselmotoren Anforderungen und Prüfverfahren. Beuth: Berlin, April 2010

\section{DANKE}

Die Ergebnisse dieser Arbeit sind mit freundlicher Unterstützung der Union zur Förderung der Oel- und Proteinpflanzen e.V. (UFOP) entstanden. Der Arbeitsgemeinschaft Qualitätsmanagement Biodiesel e.V. (AGQM) wird für die Überlassung der Analysedaten gedankt.

[4] Wilharm, T.; Stein, H.: Kurzstudie zur Evaluierung der Metall-, Phosphor- und Schwefelgehalte in Biodiesel. UFOP-Projekt Nr. 540/104, Neusäß, 2011 [5] DIN EN 14538: Erzeugnisse aus pflanzlichen und tierischen Fetten und Ölen - Fettsäure-Methylester (FAME) - Bestimmung des Ca-, K-, Mg- und $\mathrm{Na}-$ Gehaltes durch optische Emissionsspektralanalyse mit induktiv gekoppeltem Plasma (ICP). Beuth: Berlin, September 2006

[6] DIN EN 14107: Erzeugnisse aus pflanzlichen und tierischen Fetten und Ölen -Fettsäure-Methylester (FAME) - Bestimmung des Phosphorgehaltes durch Emissionsspektrometrie mit induktiv gekoppeltem Plasma (ICP). Beuth: Berlin, Oktober 2003 [7] Kern, P.; Klimczak, M.; Lucas, M.: Dieselkatalysatordesaktivierung I + II. FVV-Abschlussbericht Nr. 878, Frankfurt, 2009

[8] Klimczak, M. et al: High-throughput study of the effects of inorganic additives and poisons on NH3-SCR catalysts, Part I: V205-W03/TiO2 catalysts. In: Applied Catalysis B: Environmental 95 (2010), S. 39-47

[9] Kern, P. et al: High-throughput study of the effects of inorganic additives and poisons on NH3-SCR catalysts, Part II: Fe-Zeolite catalysts. In: Applied Catalysis B: Environmental 95 (2010), S. 48-56

[10] Guo, X.: Poisoning and sulfation on vanadia SCR catalyst. Brigham Young University, August 2006 [11] Winkler, M.; Knuth, H.W.: Durchführung eines Prüfstandsdauerlaufs über 500 Stunden sowie Feldtesterprobung zur Freigabe von Deutz-Common-RailMotoren in Nutzfahrzeugen Euro IV für Biodiesel. UFOP-Projekt Nr. 540/080, Abschlussbericht, 2009 [12] Winkler. M.: Dauerhaltbarkeitsuntersuchungen an Deutz-Agripower-Motoren der Emissionsstufe IIIB mit SCR-Systemen zur Freigabe von Biodiesel. UFOP-Projekt Nr. 540/103, UFOP-Fachkommission Biokraftstoffe, Berlin, 2011

[13] McCormick, R. L.; Alleman, T. L.; Ratcliff, M.; Moens, L.; Lawrence, R.: Survey of the Quality and Stability of Biodiesel and Biodiesel Blends in the United States in 2004. Technical Report NREL/ TP-540-38836, National Renewable Energy Laboratory, Colorado, USA, 2005

[14] Alleman, T. L.; Fouts, L.; McCormick, R. L.: Analysis of Biodiesel Blend Samples Collected in the United States in 2008. Technical Report NREL/ TP-540-46592, National Renewable Energy Laboratory, Colorado, USA, 2010

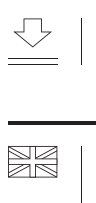

DOWNLOAD DES BEITRAGS

www.MTZonline.de

READ THE ENGLISH E-MAGAZINE

order your test issue now:

springervieweg-service@springer.com

\section{Lieferant mit Leidenschaft}

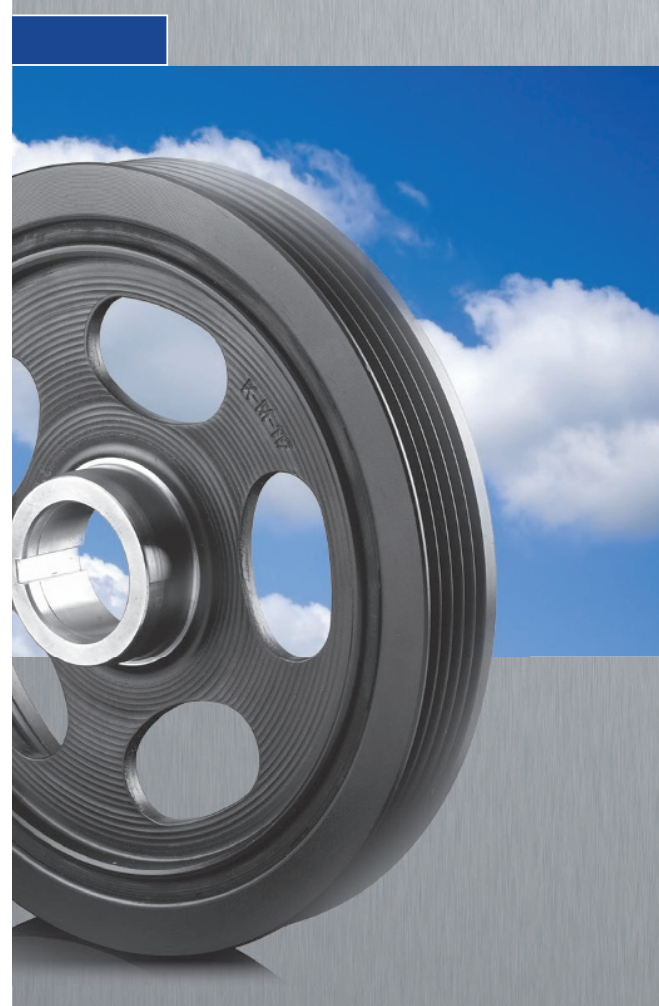

Ihre Aufgaben

sind unsere Her-

ausforderungen.

Nutzen Sie unser

Know-How in der

Auslegung und

langjährigen Pro-

jekterfahrung.

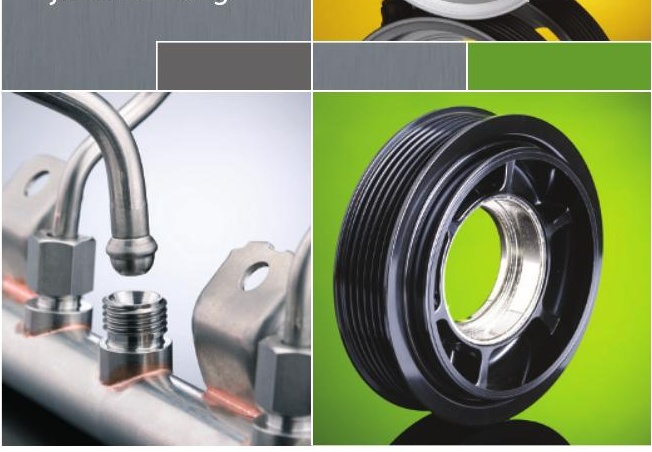

Winkelmann

Powertrain Components 